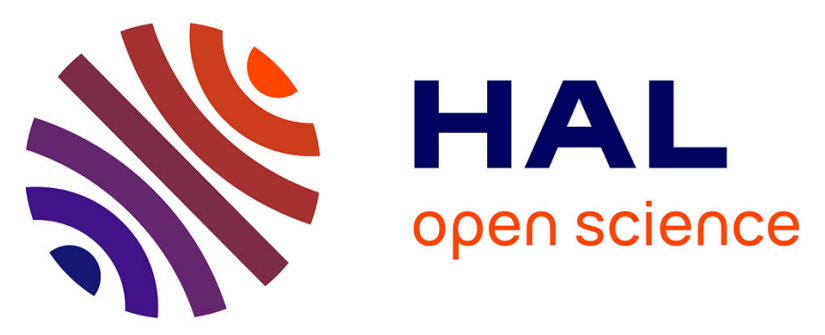

\title{
Preconditioned finite-difference frequency-domain for modeling periodic dielectric structures - comparisons with FDTD
}

\author{
Alexandre Chabory, B.P. de Hon, W.H.A Schilders, A.G. Tijhuis
}

\section{To cite this version:}

Alexandre Chabory, B.P. de Hon, W.H.A Schilders, A.G. Tijhuis. Preconditioned finite-difference frequency-domain for modeling periodic dielectric structures - comparisons with FDTD. EuMC 2008, 38th European Microwave Conference, Oct 2008, Amsterdam, Netherlands. pp 829-832, 10.1109/EUMC.2008.4751581 . hal-01022228

\section{HAL Id: hal-01022228 \\ https://hal-enac.archives-ouvertes.fr/hal-01022228}

Submitted on 23 Sep 2014

HAL is a multi-disciplinary open access archive for the deposit and dissemination of scientific research documents, whether they are published or not. The documents may come from teaching and research institutions in France or abroad, or from public or private research centers.
L'archive ouverte pluridisciplinaire HAL, est destinée au dépôt et à la diffusion de documents scientifiques de niveau recherche, publiés ou non, émanant des établissements d'enseignement et de recherche français ou étrangers, des laboratoires publics ou privés. 


\title{
Preconditioned Finite-Difference Frequency-Domain for Modelling Periodic Dielectric Structures - Comparisons with FDTD
}

\author{
A. Chabory ${ }^{\# * 1}$, B.P. de Hon ${ }^{\# 2}$, W.H.A. Schilders ${ }^{\dagger 3}$, A.G. Tijhuis ${ }^{\# 4}$ \\ \# Department of Electrical Engineering, Eindhoven University of Technology \\ P.O. Box 513, 5600MB Eindhoven, the Netherlands \\ ${ }^{2}$ B.P.d.Hon@tue.nl \\ ${ }^{4}$ A.G.Tijhuis@tue.nl \\ ${ }^{\dagger}$ Department of Mathematics and Computer Science, Eindhoven University of Technology \\ P.O. Box 513, 5600MB Eindhoven, the Netherlands \\ ${ }^{3}$ W.H.A.Schilders@tue.nl \\ ${ }^{*}$ Department of Electronics, ENAC \\ 7 avenue Edouard-Belin BP 54005, 31055 Toulouse cedex 4, France \\ 1alexandre.chabory@recherche.enac.fr
}

\begin{abstract}
Finite-difference techniques are very popular and versatile numerical tools in computational electromagnetics. In this paper, we propose a preconditioned finite-difference frequency-domain method (FDFD) to model periodic structures in $2 \mathrm{D}$ and 3D. The preconditioner follows from a modal decoupling approximation. Its use involves discrete Fourier transforms (via FFTs). We have set FDFD against an FDTD package for a typical test case, with identical spatial grids. We have observed that computation times for a full frequency sweep are comparable, while the accuracy is slightly better with FDFD.
\end{abstract}

\section{INTRODUCTION}

Finite-difference methods are well-known numerical tools in computational electromagnetics. Among them, the finitedifference time-domain (FDTD) method comprises an explicit time-stepping numerical scheme (Yee's scheme) that is very popular and simple to implement [1], [2]. Nevertheless, to model infinite periodic structures, difficulties arise in time domain methods from the implementation of periodic boundary conditions under oblique incidence (Bloch-wave boundary conditions). Harms et al., have proposed the sinecosine method to deal with this problem [3]. Further, time domain approaches are not immediately suitable (if at all) for dispersive objects, for dispersive environments, or for applications where the interest is in the time-harmonic response of a system. In such configurations, one would instead prefer a frequency-domain approach, such as the finite-difference frequency-domain (FDFD). However, for FDFD it is required to solve a large complex linear system. Krylov-subspace iterative methods are generally employed as solvers. To achieve acceptable convergence rates, they have to be accompanied by a preconditioner. In the context of 2D [4] and 3D [5] metallic waveguides, we have developed preconditioners based on fast trigonometric transforms.

In this article, we present the principles of fast-transform preconditioned FDFD for application to 2D and 3D periodic structures. Then, a numerical analysis is performed in order to compare the performance of the preconditioned-FDFD technique with FDTD.

\section{PRECONDITIONED-FDFD FOR 2D DIELECTRIC PERIODIC STRUCTURES}

A. Configuration

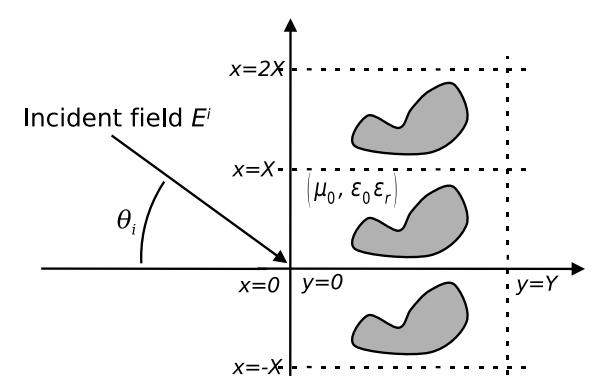

Fig. 1. 2D configuration.

In a two dimensional configuration, we consider a structure containing dielectric scatterers that is periodic with respect to $x$ (Fig. 1),

$$
\varepsilon_{r}(x+q X, y)=\varepsilon_{r}(x+q X, y), \quad \forall q \text { integer. }
$$

We assume that the structure is excited by an incident TE plane wave, i.e., $\mathbf{E}$ is oriented along $\mathbf{u}_{z}$, the unit vector in the $z$-direction. Alternatively, we may consider excitation by an electric current source such that

$$
J_{z}(x+q X, y)=J_{z}(x, y) e^{-j q k_{x i} X}, \quad \forall q \text { integer }
$$


where $k_{x i}$ is the incident wavevector along the $x$-direction. The problem then corresponds to the inhomogeneous timeharmonic Helmholtz equation

$$
\partial_{x}^{2} E_{z}+\partial_{y}^{2} E_{z}+k_{0}^{2} \varepsilon_{r} E_{z}=j k_{0} J_{z}
$$

where $k_{0}$ stands for the free-space wavenumber.

\section{B. Discretization}

On an uniform grid $(m \Delta x, n \Delta y)$, the discretization is performed using the finite-difference approximation for the spatial derivatives. This leads to

$$
\begin{aligned}
& d_{x}^{2} E_{z}[m, n]+d_{y}^{2} E_{z}[m, n] \\
& \quad+k_{0}^{2} \varepsilon_{r}[m, n] E_{z}[m, n]=j k_{0} J_{z}[m, n],
\end{aligned}
$$

where $d_{x}^{2}$ and $d_{y}^{2}$ represent the finite-difference approximations of $\partial_{x}^{2}$ and $\partial_{y}^{2}$, respectively.

\section{Boundary conditions}

In the $x$-direction, the domain can naturally be restricted to one cell of the structure $(0 \leq x \leq X)$. On the boundaries at $x=0$ and $x=X$ (corresponding to $m=0$ and $m=M$ ), the following Bloch-wave boundary conditions must be imposed

$$
E_{z}(X, y)=E_{z}(0, y) e^{-j k_{x i} X}
$$

In the $y$-direction, the physical domain is unbounded. However, to end up with a linear system of finite size, the extent of the computational domain has to be limited to the section where the sources and scatterers are located by placing two artificial boundaries at $y=0$ and $y=Y$ (i.e. $n=0$ and $n=N$ ) on which absorbing boundary conditions are required. This could be realised by adding PML layers [6], but as in [4] and [5], a computationally-efficient and yet exact approach can be employed.

For each $n$, we first note that the transverse electric field $E_{z}[m, n]$ can be represented as a linear combination of discrete modal voltages $V[p, n]$, where $p$ stands for the modal order. The transformation $U_{v}^{H}: E_{z} \rightarrow V$ may be expressed as

$$
U_{v}^{H}=F \Lambda_{i}
$$

in which $F$ is the discrete Fourier transform of size $M$, and $\Lambda_{i}$ introduces phase shifts for non-zero $k_{x i}$. If no scatterers or sources are present near the boundaries $y=0$ and $y=Y$, exact discrete absorbing boundary conditions are obtained by imposing that each modal voltage should propagate or attenuate away from the computational domain. This can be formulated as

$$
\begin{aligned}
\left.E_{z}\right|_{n=0} & =\left.U_{v} \Gamma U_{v}^{H} E_{z}\right|_{n=1}, \\
\left.E_{z}\right|_{n=N} & =\left.U_{v} \Gamma U_{v}^{H} E_{z}\right|_{n=N-1},
\end{aligned}
$$

where $\Gamma$ is a diagonal operator containing the propagation or attenuation of modes that have travelled a distance $\Delta y$.

\section{Linear system}

From (4), (5) and (7), a linear system $A u=b$ is obtained. The unknown $u$ and the right-hand-side $b$ are vectors containing the $E_{z}$ field and the source term, respectively. Within the Krylov-subspace iterative solver, one iteration mainly requires the computation of a few matrix-vector products. Thus, if FFTs are used for the $U_{v}$ transforms in (7), the cost of one iteration will be of order $O(M N+M \log M)$, which is very efficient.

\section{E. Fast-transform based preconditioner}

The preconditioner $A_{0}$ is obtained from a physical approximation of the original linear operator $A$. As in [4] and [5], it is constructed so that all the modal voltages are decoupled. This yields an approximation of the actual configuration only in the inhomogeneous regions where the scatterers are located. Mathematically, it is defined as the optimal approximation of $A$ with respect to the Frobenius norm such that the modal voltages are decoupled. The decoupling replaces the initial 2D problem by $N-1$ single-mode uncoupled 1D problems that can be associated with tri-diagonal systems of size $M$. Computation time can be reduced by employing $A_{0}$ as a preconditioner only if $A_{0} w=d$ can be solved rapidly for any vector $d$. Such a rapid solver exists, and consists of three steps:

1) Compute the modal source term from $d$ using $U_{v}^{H}$,

2) Solve the single-mode problems (tri-diagonal systems),

3) Compute the solution using $U_{v}$.

Thus the complexity of solving $A_{0} w=d$ is of order $M N(\log M+\log N)$ which is moderate compared to the cost of the matrix-vector product $A v$.

\section{EXTENSION TO 3D PERIODIC STRUCTURES}

A similar approach can be developed for 3D dielectric structures that are periodic with respect to the $x$ - and $y$-directions. On a regular lattice, the finite-difference discretization of the time-harmonic normalised Maxwell equations is given by [5]

$$
\left[\begin{array}{cc}
j k_{0} \varepsilon_{r} & -\mathbf{d} \times \\
\tilde{\mathbf{d}} \times & j k_{0}
\end{array}\right]\left[\begin{array}{l}
\mathbf{e} \\
\mathbf{h}
\end{array}\right]=-\left[\begin{array}{l}
\mathbf{j} \\
\mathbf{0}
\end{array}\right],
$$

in which $\mathbf{d} \times$ and $\tilde{\mathbf{d}} \times$ are finite-difference approximations of the curl operator. Further, $(\mathbf{e}, \mathbf{h})$ and $\mathbf{j}$ are normalised fields and electric sources, respectively. Each component $u$ of the fields must respect the following Bloch-wave boundary conditions,

$$
\begin{aligned}
& u(X, y)=u(0, y) e^{-j k_{x i} X} \\
& u(x, Y)=u(x, X) e^{-j k_{y i} Y},
\end{aligned}
$$

where $(X, Y)$ are the size of one cell, and $\left(k_{x i}, k_{y i}\right)$ are the transverse components of the incident wavevector. The absorbing boundary conditions and the preconditioner are defined in line with the principles explained above. Note that the expression of the discrete modal representation now has to include both discrete modal voltages and currents, as for the 3D metallic waveguide [5]. 


\section{NUMERICAL COMPARISONS WITH FDTD}

\section{A. Test case}

As a test case for comparisons with FDTD, we consider a 2D electromagnetic band gap (EBG) material. Each cell of the structure is of size $40 \mathrm{~mm} \times 240 \mathrm{~mm}$ and contains 6 dielectric scatterers of relative permittivity $\varepsilon_{r}=6$ (Fig. 2). We examine the ability of both numerical methods to compute the transmission coefficient of this material at normal incidence and for 200 frequency points between 0 and $6 \mathrm{GHz}$.

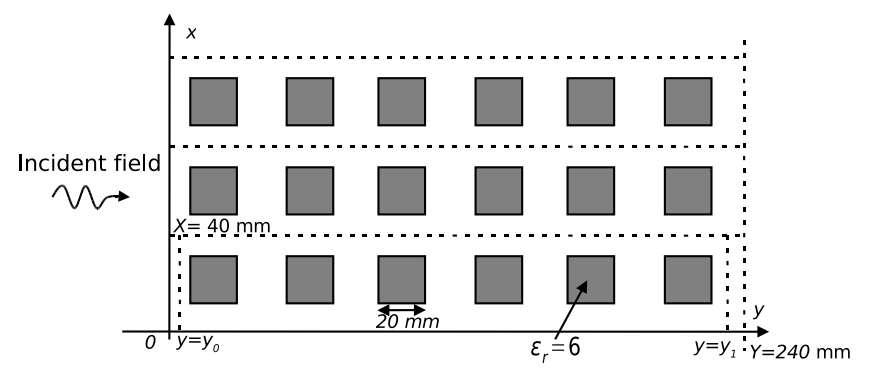

Fig. 2. Test case configuration.

\section{B. FDTD simulation}

FDTD simulations are performed using the freely available software package Meep [7]. In this software, PML layers [6] must be inserted to model the absorbing boundaries at $y=0$ and $y=Y$. The incident plane wave is generated by an electric current source placed at $y=y_{0}$ which expression is given by

$$
J_{z}(x, t)=\cos \left(2 \pi f_{0} t\right) e^{-t^{2} / T_{w}^{2}}
$$

The parameters $f_{0}$ and $T_{w}$ are chosen so as to cover a wide frequency range. The frequency response is computed from an FFT of the time-domain field obtained at $y=y_{1}$. The spatial grid size is determined so that the grid steps along $x$ and $y$ are the same. We choose $\mathrm{N}=6 \mathrm{M}=192$ which corresponds to having more than 16 points per local wavelength in all the frequency range.

\section{Preconditioned-FDFD simulation}

To generate the incident plane wave in the frequency domain, we place a constant electric current source at $y=y_{0}$. The Krylov-subspace iterative method we use is BiCGstab(2) [8]. The stopping criterion is defined so that the norm of the residual $r_{i}=b-A u_{i}$ is reduced by a factor of $10^{-6}$ with respect to the norm of $b$ after the last iteration. To provide a good initial guess to the iterative solver, at each frequency step, we take into account the results corresponding to the 3 preceding frequencies via the "marching on in anything" method [9].

\section{Results and comparisons}

In Fig. 3, we depict the amplitude of the transmission coefficient of the material. We observe 3 band-gaps centred at 2.5, 4.5 and $6 \mathrm{GHz}$. We note a good agreement between both numerical methods. Nevertheless, if we look closer at the values found for low and high frequencies, significant differences appear (Fig. 4). In these regions, FDTD gives nonphysical results (the transmission coefficients exceed $0 \mathrm{~dB}$ ). At low frequencies, errors with FDTD may be explained by the PML layers that are not thick enough to correctly absorb waves impinging the boundaries. This source of errors does not exist with the preconditioned-FDFD approach due to the exact absorbing boundary conditions (5) that are employed. At high frequencies, errors originate from the size of the grid. They can be reduced by using a finer grid, which would however yield longer computation times.

On a PC running at $2.4 \mathrm{GHz}$, results of Fig. 3 were obtained in $18.6 \mathrm{~s}$ for FDTD and in $25.6 \mathrm{~s}$ for the preconditionedFDFD method, thus in comparable computation times. In Fig. 5 , we show the evolution of computation times with respect to the grid size. We note that the speed of FDTD and of the preconditioned-FDFD method remains similar regardless of the grid size, although the FDTD scheme is explicit (and hence fast), whereas a linear system must be solved at each frequency point with the preconditioned-FDFD method. This demonstrates the efficiency of the preconditioner that we propose. For narrower frequency bands the balance will unequivocally tip in favour of FDFD.

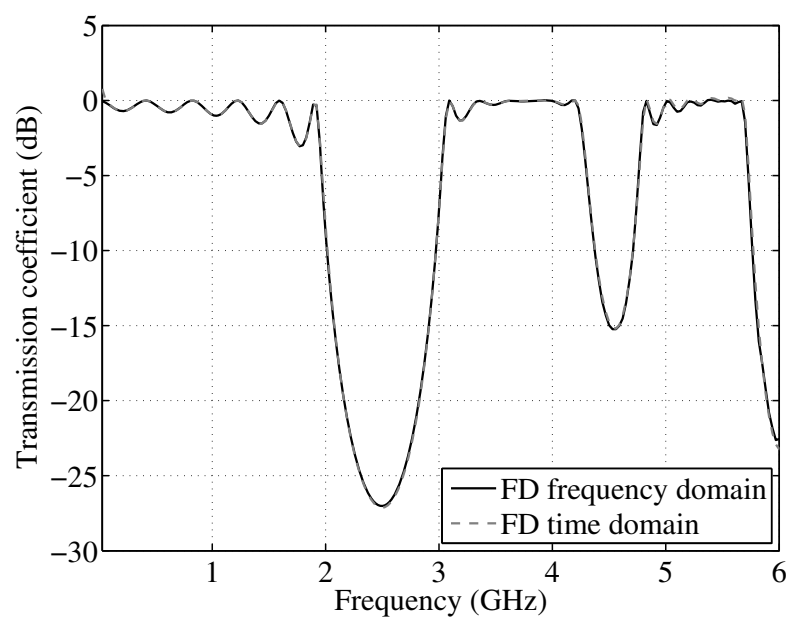

Fig. 3. Transmission coefficient (dB).

\section{CONCLUSIONS}

We have proposed a preconditioned-FDFD method to compute the fields in the presence of dielectric scatterers arranged periodically with respect to the transverse directions in $2 \mathrm{D}$ and 3D. The modal representation of the solution has been used to obtain exact discrete absorbing boundary conditions. We have exposed the principles of a preconditioner based on a physical approximation and involving FFTs. Comparisons on a test case have shown that FDFD can compete with FDTD when full frequency sweeps are required, indicating that for narrow-band applications FDFD will outperform FDTD. Moreover, general material dispersion can easily be handled 

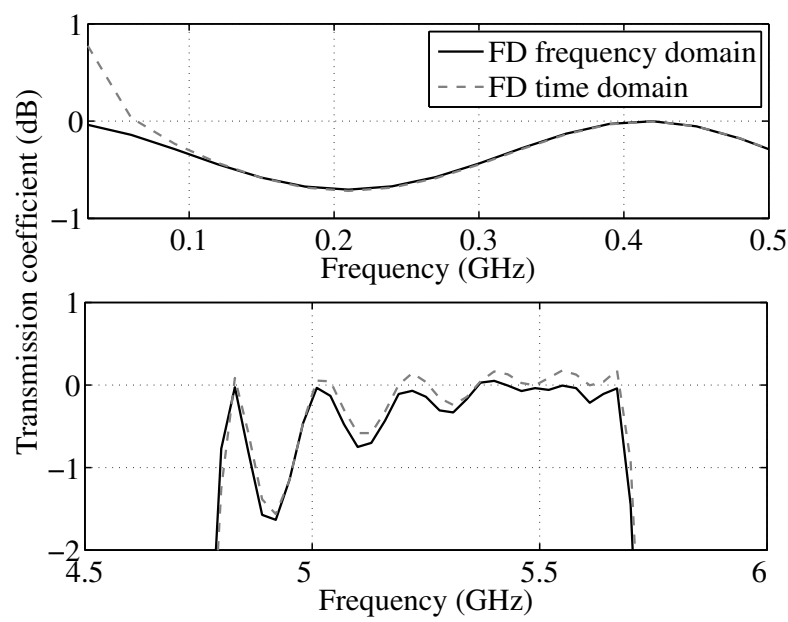

Fig. 4. Transmission coefficient $(\mathrm{dB})$ at low and high frequencies.

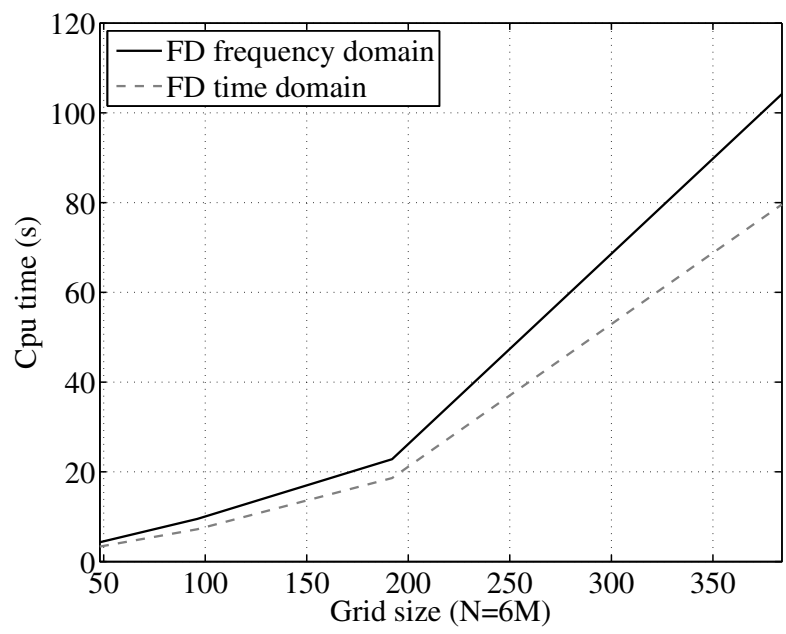

Fig. 5. Computation time with respect to the grid size $N(=6 M)$.

in the FDFD formalism. Further, oblique incidence poses no problems either.

\section{ACKNOWLEDGEMENT}

The authors wish to thank NWO for supporting this work under contract 99008541.

\section{REFERENCES}

[1] K.S. Yee, "Numerical solution of initial boundary value problems involving Maxwell's Equations in isotropic media”, IEEE trans. Antennas Propagat., vol. 14 , no. 3, pp. 302-307,1966.

[2] A.Taflove and S.C. Hagness, Computational Electrodynamics: The FiniteDifference Time-Domain Method, Artech: Norwood, MA, 2000.

[3] P. Harms, R. Mittra, W. Ko, "Implementation of the periodic boundary condition in the finite-difference time-domain algorithm for FSS structures“, IEEE trans. Antennas Propagat., vol. 42, no. 9, pp. 1317-1323, 1994.

[4] A. Chabory, B.P. de Hon, A.G. Tijhuis, "Sine transform based preconditioner for the FDFD method inside a parallel plate waveguide", PIERS (Progress In Electromagnetics Research Symposium), Tokyo, Japan, august 2006
[5] A. Chabory, B.P. de Hon, W.H.A. Schilders, A.G. Tijhuis, "Fast transform based preconditioner for 3D finite-difference in the frequency domain Application to waveguides“, ICEAA, Torino, Italy, September 2007.

[6] J.P. Berenger, "Three-dimensional perfectly matched layer for the absorption of electromagnetic waves“, Journal of Computational Physics, vol. 127, pp. 363-379, 1996.

[7] A. Farjadpour, D. Roundy, A. Rodriguez, M. Ibanescu, P. Bermel, J.D., S.G. Johnson, G. Burr, "Improving accuracy by subpixel smoothing in FDTD“, Optics Letters, vol. 31, no. 20, pp. 2972-2974, 2006.

[8] G.L.G. Sleijpen, D.R. Fokkema, "BiCGstab(ell) for linear equations involving unsymmetric matrices with complex spectrum", Electronic Transactions on Numerical Analysis, vol. 1, pp. 11-32, 1993.

[9] A.G. Tijhuis, K. Belkebir, P. Zwamborn, and A. Rubio Bretones,"Marching on in anything: Solving electromagnetic field equations with a varying parameter", Proc. Int. Conf. Electromagnetics in Advanced Applications, Torino, Italy, pp. 175-178, 1997. 